\title{
Destination Aware APU Strategy for Geographic Routing in MANET
}

\author{
Yukti \\ M.tech Student \\ Department of Computer science and engineering \\ Giani Zail Singh- Punjab Technical University Campus \\ Bathinda, Punjab, India
}

\author{
Abhilasha jain \\ Associate professor \\ Department of Computer science and engineering \\ Giani Zail Singh- Punjab Technical University Campus \\ Bathinda, Punjab, India
}

\begin{abstract}
In this paper, we have explained the Enhanced Adaptive Position Update strategy for geographic routing in mobile ad hoc network In Adaptive Position Update strategy, there are two techniques: Mobility prediction rule and On-demand learning rule. Proposed system is based on the destination aware routing in which path to transfer the data over the network is based on the distance from highly stable node to the destination node. Results of the proposed system are compared with Periodic Beaconing on the basis of packet delivery ratio, beacon overhead, energy consumption. Experiment results show a high improvement in results on the parameters energy consumption, packet delivery ratio and beacon overhead. Proposed work is implemented on the NS2 (Network Simulator) Environment to perform experiments.
\end{abstract}

Keywords: APU, Enhanced APU, MANET, Destination aware routing.

\section{INTRODUCTION}

MANET is self-configured Mobile Ad hoc network of mobile nodes connected via random topology. The main issue in MANET is the selection of best path between any two nodes [1]. Routing in MANET is to find the efficient path to transfer the data from source to destination.

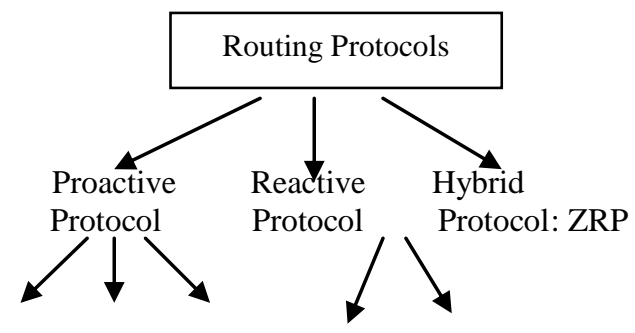

DSDV WRP OLSR DSR AODV

Figure 1. Categorization of routing protocols

Main protocols used for routing in MANET are categorized as Proactive protocols, Reactive protocols and hybrid protocols. Proactive protocols are those which are derived from past study and information is maintained in the form of tables so also called table-driven (eg. DSDV, WRP, OLSR). In Reactive protocols, instead of past approaches the nodes send the data packets to the destination on-demand (eg. DSR, AODV).In hybrid protocols, the combination of both the reactive and proactive protocols, this protocol includes ZRP (Zone Routing Protocol) [2]. These protocols are briefly explained as: In DSDV, enhancement is done on Bellman-ford Routing Algorithm by making it loop-free. In this, each node maintain a routing table which contains the entries of all the devices in network and table is updated via periodically broadcast routing messages to its neighbor. In WRP, which also resembles the DSDV protocol and its main idea is to find the shortest distance to every node and it is basically a path-finding algorithm with exception that it counters the count-to-infinity problem by forcing each node to perform consistency checks of predecessor information reported by all its neighbors. This eliminates looping situations and enables faster route convergence when a link failure occurs. In OLSR, point-to-point approach that employs a link-state mechanism known as multipoint relying. Two ways are there: by reducing the size of control packets or by reducing the number of links used in forwarding the packets. The methodology used is first the analysis of changes in the node's neighborhood called neighbor sensing then find the best path to every destination using this information and store it in table called routing table. Then the best route for destination is computed via shortest-path algorithm. In DSR fig.2, the unique feature is that it doesn't use periodic updates. In this, basically two things happen: route discovery and route maintenance. First route is discovered on-demand i.e. it finds the route when necessary for that sender determines the complete series of the nodes from source to destination and lists this route in packet header and save it in cache. If source wants to send data it first check the cache if there is entry in cache for that destination, I transfer the packet otherwise it broadcast a route request to all its neighbors and wait, then the node having the route information send the request reply to source and sender send the packets to discovered route and also make entry in cache for further use.

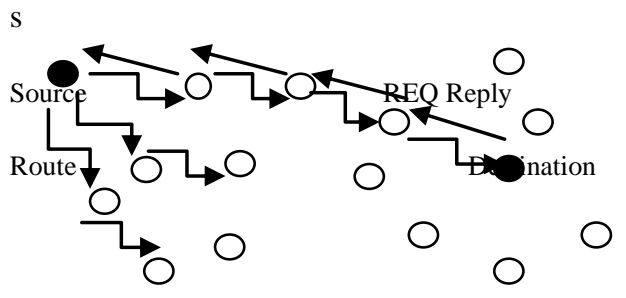

Figure 2. DSR protocol 
In AODV, the concept of DSR is used in addition; it uses the hop-to-hop routing, sequence numbers and periodic beacons from DSDV. It removes the drawback of WRP proactive protocol and finds the route only if required. The main problems in AODV are: i) gradual increase in sequence numbers and ii) decrease in hop count and It is not secure. In ZRP, advantages of both proactive and reactive protocols are mixed. It works in two parts of which first uses the table-driven approach to speed up communication and later uses the ondemand approach to avoid unnecessary communication [3].

Further comes the concept of geographic routing which is widely used in Mobile ad hoc networks due to its simplicity and scalability and has low overhead compared to other protocols. Geographic routing based on assumption that every node is aware of its location in the network (location information generated via GPS and schemes explained in [16]). Based on this assumption, various protocols are derived and worked on: DREAM, LAR, GPSR. These routing protocols are explained as follows: In DREAM, nodes forwards data packets to all the neighbors that lie in direction of destination and every node updates every other node about its location. In LAR, it is assumed that every node is aware of its location but can't predict location of other nodes. It basically reduces the flooding overhead. Two LAR schemes are proposed is sending node is only within request zone and outer area is not addressed and second is the nodes always send the packets to node closer to the destination [5].In GPSR, nodes forwards the data packets based on distance. It comprises two techniques: greedy forwarding and face routing. Till the destination reached, the forwarding is carried out on greedy basis by selecting the closest node to destination. If this method doesn't work then face routing is used. After the node is in transmission range then again greedy forwarding is used [6].

The remaining part of the paper is organized as: In Section 2, we discuss related work or the literature survey then in Section 3 the proposed scheme and Section 4 signifies the results and Section 5 concludes the paper.

\section{RELATED WORK (LITERATURE SURVEY)}

Many papers and articles are proposed on Mobile ad hoc Network and the protocols defining the routing of packets among network. There are Distance routing protocols and Location based routing protocols and further we are using new technique for the same. According to [1], GeRaF is the forwarding technique based on the assumption that nodes has a medium to detect their location and position of final destination and transmitting node is included in the forwarding message.

\section{Problems with the existing system}

Problems in Position updates in network are costly in many ways.

1. Each update in Position in a given network consumes node energy, bandwidth of wireless channel, and the risk of packet collision increases correspondingly at the medium access control (MAC) layer.

2. Packet collisions in the network cause packet loss which again decreases the routing performance which results in difficulty to evaluate local topology for transmission. It results in a heavy loss due this factor beacon lost and a lost beacon cannot be retransmitted.

3. A lost data packet does get retransmitted as a result end-to-end delay increases.

In the existing system, Periodical beacon broadcasting is used to exchange the neighbor's location that results in degradation in performance, increases packet loss and results in longer end to end delay. Hence a more robust update policy is required to be discovered to overcome all these types of the costs.

\section{PROPOSED WORK}

An Enhanced APU strategy in the proposed system use destination aware routing with minimum distance dynamically adjusts the beacon update intervals based on the minimum distance from source to destination, mobility dynamics of the nodes and the forwarding patterns in the network.

The beacons transmitted by the nodes contain their current position and speed. Nodes estimate their positions periodically by employing linear kinematic equations based on the parameters announced in the last announced beacon. If the predicted location is different from the actual location, a new beacon is broadcast to inform the neighbors about changes in the node's mobility characteristics.

An accurate representation of the local topology is particularly desired at those nodes that are responsible for forwarding packets. Hence, APU seeks to increase the frequency of beacon updates at those nodes that overhear data packet transmissions. As a result, nodes involved in forwarding packets can build an enriched view of the local topology.

Algorithm for selection of forwarder

Step1: Find distance $[\mathrm{d}(\mathrm{t})]$ of each neighbor from source at time $\mathrm{T}$

Step2: Find distance $[\mathrm{d}(\mathrm{t}+\mathrm{T})]$ of each neighbor from source at time $(\mathrm{T}+\mathrm{t})$

Step3: If $\{[\mathrm{d}(\mathrm{t}+\mathrm{T})] \sim[\mathrm{d}(\mathrm{t})]<$ Threshold $\} \rightarrow$ Select the neighbor as high stable link

Step4: Find distance $\mathrm{D}_{\text {des }}$ between destination and the node having high stable link

Step5: Link having minimum $\mathrm{D}_{\text {des }}$ is selected as next hop

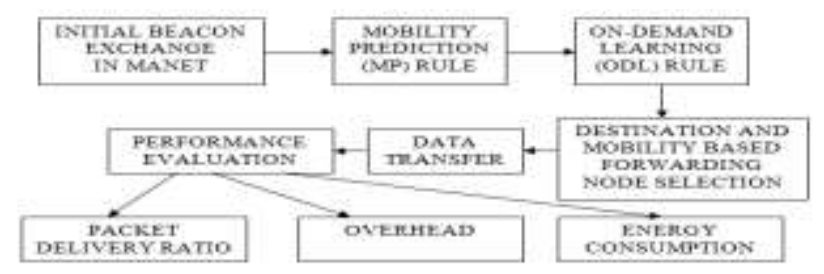

Figure 3. Block Diagram representing the functions in the proposed APU system 
In the above fig. 3 different modules of the proposed APU system to update the position of beacon are represented.

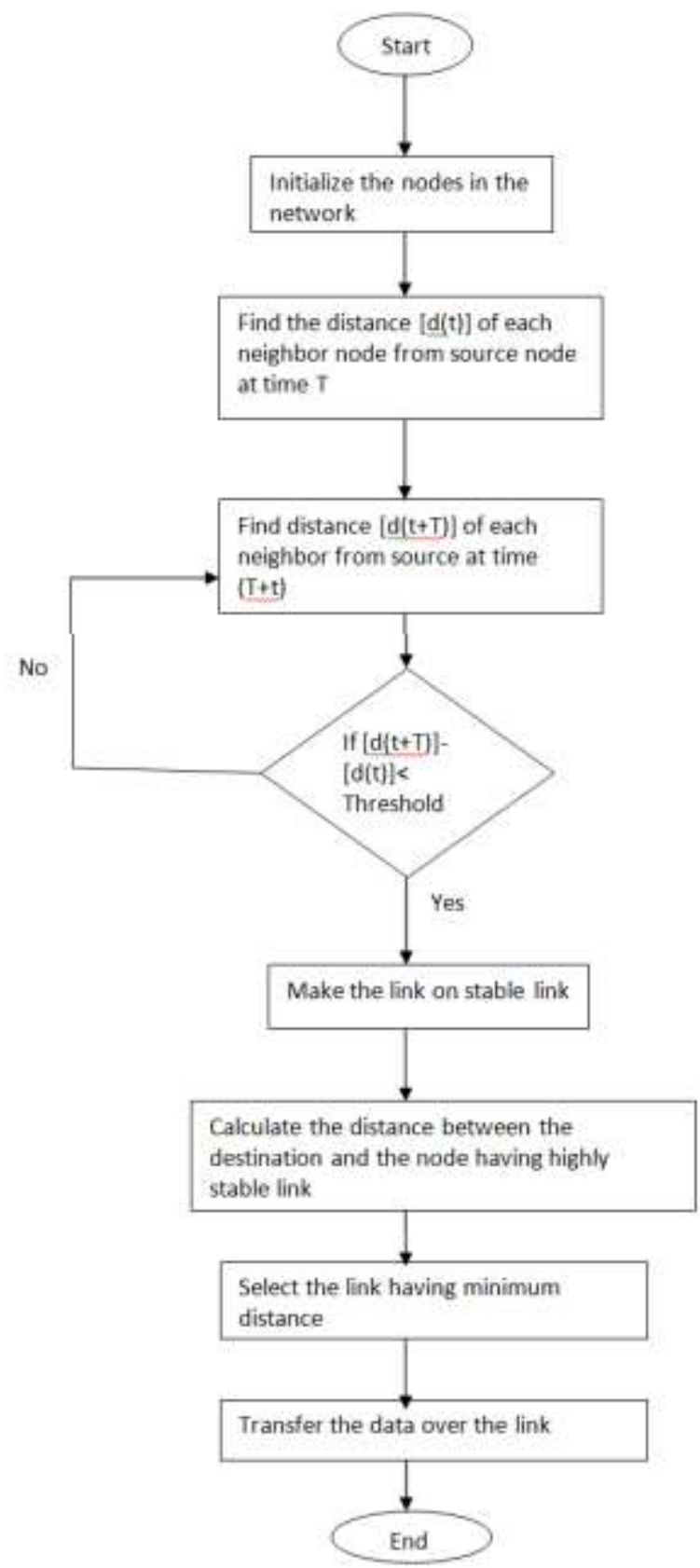

Figure 4. Flowchart for the proposed APU system

The Flowchart (fig.4) represents the methdology of the work done.Initially the nodes in the network have been deployed in such a way that all the nodes are location aware. All location aware nodes broadcast beacons to their neighbor nodes to calculate the distance from source at time T. After that the distance at time $(\mathrm{t}+\mathrm{T})$ is calculated. If the difference between the calculated distances is less than threshold then it is considered as stable link. Select the link having the minimum distance by calculating the distance between destination node and highly stable link and then data can be transferred over the network.

\section{RESULTS AND DISCUSSION}

Proposed APUsystem is evaluated on the following parameters:

\subsection{PDR (Packets Delivery Ratio): PDR is the}

proportion to the total amount of packets reached the receiver and amount of packet sent by source. If the amount of malicious node increases, PDR decreases. The higher mobility of nodes causes PDR to decrease.
PDR $(\%)=$ Number of packets successfully delivered to destination
Number of packets generated by source node

4.2 Energy Consumption: It is the amount of energy consumed by the sensors for the data transmission over the network

Energy Consumption $=$ Sum of energy consumed by each sensor.

4.3 Overhead: It is defined as the number of messages involved in beacon update process.

Overhead $=$ No. of messages involved in beacon update process

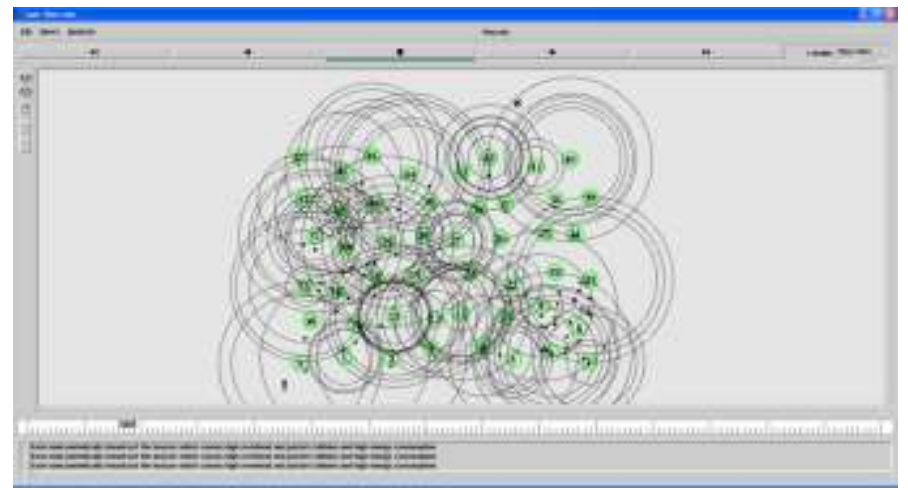

Figure 5. Implementation of the proposed APU system in NS2 environment

Above snapshot (fig.5)shows each node periodically broadcast the beacon which causes high overhead and packet collision and high energy consumption. 


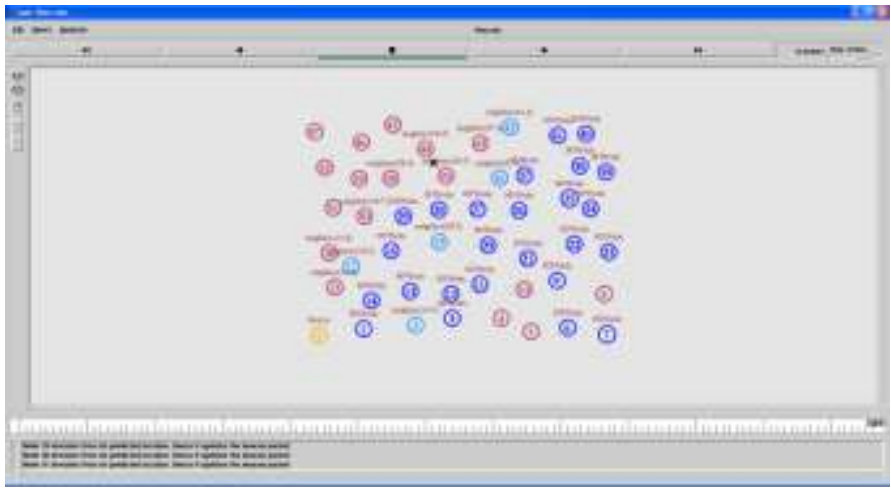

Figure 6. Node selection in proposed APU system

In given fig.6 Nodes in blue color are MP nodes which are having the actual location with larger difference from its predicted location. Hence MP nodes update the beacon packet. Deviation threshold is fixed as $60 \mathrm{~m}$. If there exists difference between actual locations and predicted of a node is greater than $60 \mathrm{~m}$ then beacon packet is sent by the node.

Table 1. Energy Consumption versus Time

\begin{tabular}{|c|c|c|}
\hline & Existing & Proposed \\
\hline 0 & 0 & 0 \\
\hline 2 & 75 & 40 \\
\hline 4 & 150 & 75 \\
\hline 6 & 225 & 120 \\
\hline 8 & 310 & 160 \\
\hline 10 & 380 & 190 \\
\hline 12 & 460 & 230 \\
\hline 14 & 540 & 275 \\
\hline
\end{tabular}

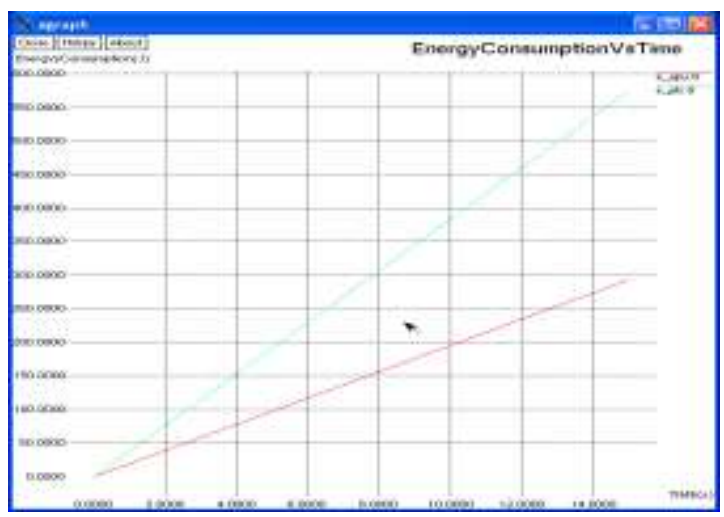

Figure 7. graph showing energy consumption in existing scheme and proposed APU scheme
Given fig.7 shows the energy consumption in existing scheme and proposed APU scheme and the table1 showing the values of graphs.

Energy consumption in existing scheme is high compared to proposed APU since periodic beacon causes high energy consumption in the nodes. APU saves energy by avoiding unnecessary beacon update and do the beacon update adaptively.

Table 2. Beacon Overhead versus Time

\begin{tabular}{|c|c|c|}
\hline & Existing & Proposed \\
\hline 0 & 0 & 0 \\
\hline 2 & 14 & 0 \\
\hline 4 & 27 & 1 \\
\hline 6 & 40 & 1 \\
\hline 8 & 54 & 2 \\
\hline 10 & 68 & 3 \\
\hline 12 & 81 & 3 \\
\hline 14 & 95 & 4 \\
\hline
\end{tabular}

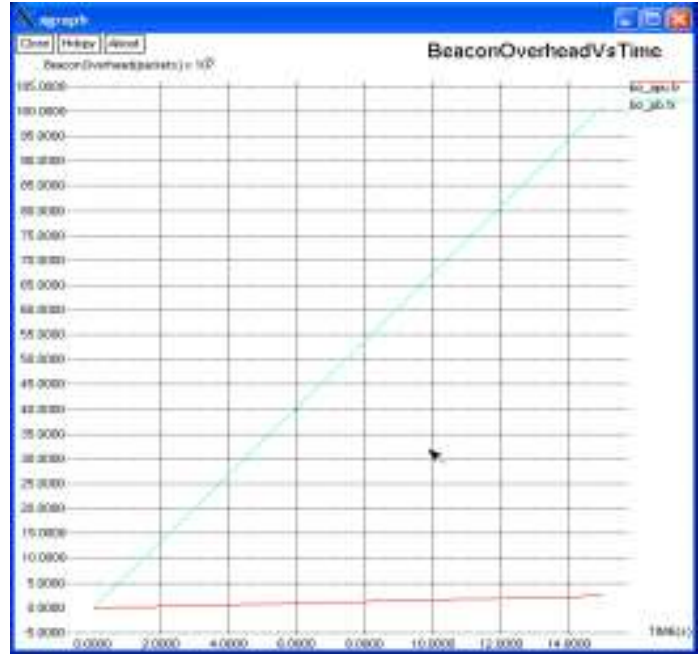

Figure 8. graph showing beacon overhead in existing scheme and proposed APU scheme

Given fig. 8 shows the beacon overhead in existing scheme and proposed APU scheme and the table 2 showing the values of graphs.

Beacon overhead in existing scheme is high compared to proposed APU due to periodic beacon. APU reduces the beacon overhead by avoiding unnecessary beacon update and only does the beacon update process adaptively. 
Table 3. Packet Delivery Ratio versus Time

\begin{tabular}{|c|c|c|}
\hline & Existing & Proposed \\
\hline 0 & 0 & 0 \\
\hline 2 & 5 & 12 \\
\hline 4 & 9 & 23 \\
\hline 6 & 14 & 35 \\
\hline 8 & 19 & 47 \\
\hline 10 & 23 & 59 \\
\hline 12 & 27 & 72 \\
\hline 14 & 37 & 83 \\
\hline
\end{tabular}

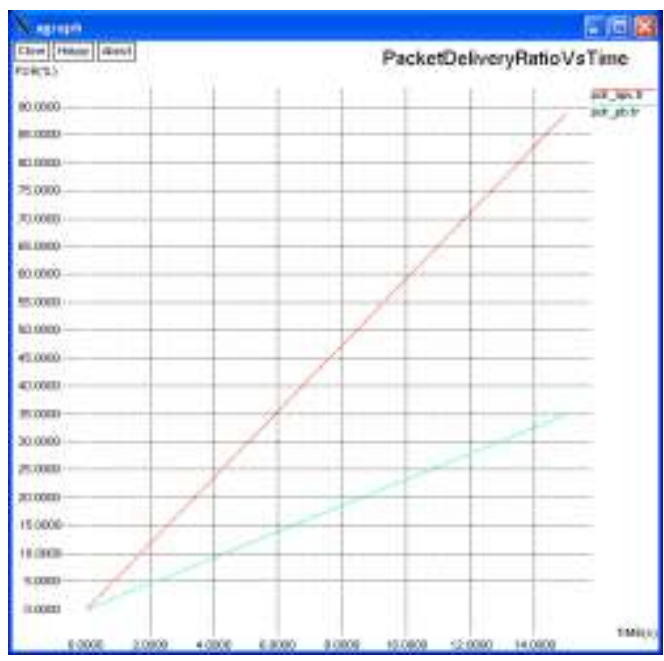

Figure 9. graph showing beacon overhead in existing scheme and proposed APU scheme

Given fig. 9 shows the packet delivery ratio in existing scheme and proposed APU scheme and the table 3 showing the values of graphs.

Packet delivery ratio of proposed APU is high compared to existing scheme. Since network traffic in APU is reduced due to adaptive beacon update instead of periodic beacons in the case of periodic beacon scheme. In PB data gets dropped due to high traffic in the network.

\section{CONCLUSION AND FUTURE WORK}

In this paper, the need to adapt the beacon update is identified and the corresponding policy is employed in geographic routing protocols to the node mobility dynamics and the traffic load. The Adaptive Position Update (APU) strategy is proposed to address these problems. The APU scheme employs two mutually exclusive rules. The MP rule uses mobility prediction to estimate the accuracy of the location estimate and adapts the beacon update interval accordingly, instead of using periodic beaconing. The ODL rule allows nodes along the data forwarding path to maintain an accurate view of the local topology by exchanging beacons in response to data packets that are overheard from new neighbors. Performance of APU is evaluated using extensive NS-2 simulations for varying node speeds and traffic load. Proposed APU strategy leads to cost reduction in beacon update due to destination aware routing and increase in performance over existing system. Results indicate that the APU strategy generates less or similar amount of beacon overhead as other beaconing schemes but achieve better packet delivery ratio, less overhead and energy consumption.

Future work will be the exploring the new techniques to the proposed work to reduce the overhead and energy consumption further in the network.

\section{REFERENCES}

[1]Sangeeta, Kirti Singh(2011),"Energy Efficient Routing in MANET using OLSR", International Journal on Computer Science and Engineering, vol. 3, no. 4.

[2] Smita Singh, Shradha Singh, Soniya jain, S.R. Birdar(2012), "Comparison and study of AOMDV and DSDV Routing Protocols in MANET using NS-2", International Journal on Computer Science and Engineering, vol. 4, no. 3.

[3]Anuj K.Gupta, Harsh Sadawarti, Anil K.Verma(2011), "Review of Various Routing Protocols for MANETs", International Journal of Information and Electronics Engineering, vol. 1, no. 3.

[4]Gaurav Sachan, D.K.Sharma, Karishma Tyagi, Abhimanyu Parsad(2013), "Enhanced Energy Aware geographic Routing Protocol in MANET: A Review", International Journal of Modern Engineering Research,vol. 3, pp. 845-848, Issue 2.

[5]Divya Wadhwa, Deepika, Vimmi Kochher, Rajesh Kumar Tyagi(2014), "A Review of Comparison of Geographic Routing Protocols in Mobile Adhoc Network", Advance in Electronic and Electric Engineering, vol.4, pp. 51-58, no.1.

[6]Varun G Menon, Joe Prathap P M(2013)," Performance Analysis of Geographic Routing Protocol in Highly Mobile Ad hoc Network", Journal of Theoretical and Applied Information Technology, vol. 54, no. 1 .

[7]Michele Zorzi, Ramesh R. Rao(2003), “Geographic Random Forwarding $(\mathrm{GeRaF})$ for ad hoc and sensor networks: energy and latency performance", IEEE Transactions on Mobile Computing, vol. 2 , no. 4 . 
[8]Quan Jun Chen, Salil S. Kanhere, Mahbub Hassan, Kun-Chan Lan(2005), "Adaptive Position Update in Geographic Routing", School of Computer Science and Engineering, The University of New South Wales, Sydney, Australia.

[9]Dongjin Son, Ahmed Helmy, Bhaskar Krishnamachari,"The Effect of Mobility-induced Location Errors on Geographic Routing in Mobile Ad Hoc and Sensor Networks: Analysis and Improvement using Mobility Prediction”, Department of Electrical Engineering,University of Southern California, 90089.

[10] Pasupuleti Neelima, K.Bhargavi(2014), "Beaconing Strategy for Geographic Routing in Mobile Ad Hoc Network",International Journal of Innovative Research in Computer and Communication Engineering, Vol. 2, Issue 7.

[11]Ravi Kumar Poluru, T. Sunil Kumar Reddy(2014), D.Nagaraju, "Updating the Routing Position in Ad hoc Networks by using Adaptive Position", International Journal of Computer Trends and Technology (IJCTT), Vol. 10 No. 3.

[12]B. Karp and H. T. Kung., "GPSR: Greedy Perimeter Stateless Routing for Wireless Networks", in Proceedings of ACM Mobicom 2000, pp. 243-254, Boston, MA, USA,2000.

[13]P. Casari, M. Nati, C. Petrioli and M.Zorzi(2007), "Efficient Non Planar Routing around Dead Ends in Sparse Topologies using Random Forwarding", in Proceedings of IEEE ICC, pp.3122-3129. Scotland,UK.

[14]Y. Kim, R. Govindan, B. Karp and S. Shenker(2005), "Geographic routing made practical", in Proceedings of the 2nd Conference on Symposium on Networked Systems Design and Implementation, pp. 217-230, Berkeley, CA, USA.

[15]Quanjun Chen, Salil S. Kanhere, Mahbub Hassan(2013), "Adaptive Position Update for Geographic Routing in Mobile Ad Hoc Networks", IEEE Transactions on Mobile Computing, vol. 12, no. 3 .

[16]J. Hightower and G. Borriello(2001), "Location System for Ubiquitous Computing", IEEE computer, vol. 34, pp. 57-66, no. 8.

[17]Paolo Casari, Michele Nati, Chiara Petrioli, Michele Zorzi(2007), "Efficient Non-Planar Routing around Dead Ends in Sparse Topologies using Random Forwarding", IEEE Communications Society, pp. 3122-3129. 